\title{
Anti-PD-1 and Novel Combinations in the Treatment of Melanoma-An Update
}

\author{
Frank Friedrich Gellrich 1,2,*(D), Marc Schmitz ${ }^{3,4}$, Stefan Beissert ${ }^{1,2}$ and Friedegund Meier ${ }^{1,2}$ \\ 1 Department of Dermatology, University Hospital Carl Gustav Carus, TU Dresden, 01307 Dresden, Germany; \\ Stefan.Beissert@uniklinikum-dresden.de (S.B.); Friedegund.Meier@uniklinikum-dresden.de (F.M) \\ 2 Skin Cancer Center at the University Cancer Centre Dresden and National Center for Tumor Diseases, \\ 01307 Dresden, Germany \\ 3 Institute of Immunology, Medical Faculty Carl Gustav Carus, TU Dresden, Fetscherstraße 74, 01307 Dresden, \\ Germany; mschmitz@mail.zih.tu-dresden.de \\ 4 National Centre for Tumor Diseases, University Hospital Carl Gustav Carus, TU Dresden, Fetscherstraße 74, \\ 01307 Dresden, Germany \\ * Correspondence: frankfriedrich.gellrich@uniklinikum-dresden.de; Tel.: +49(0)351-458-2497
}

Received: 21 December 2019; Accepted: 7 January 2020; Published: 14 January 2020

\begin{abstract}
Until recently, distant metastatic melanoma was considered refractory to systemic therapy. A better understanding of the interactions between tumors and the immune system and the mechanisms of regulation of T-cells led to the development of immune checkpoint inhibitors. This review summarizes the current novel data on the treatment of metastatic melanoma with anti-programmed cell death protein 1 (PD-1) antibodies and anti-PD-1-based combination regimens, including clinical trials presented at major conference meetings. Immune checkpoint inhibitors, in particular anti-PD-1 antibodies such as pembrolizumab and nivolumab and the combination of nivolumab with the anti-cytotoxic T-lymphocyte-associated protein 4 (CTLA-4) antibody ipilimumab can achieve long-term survival for patients with metastatic melanoma. The anti-PD-1 antibodies nivolumab and pembrolizumab were also approved for adjuvant treatment of patients with resected metastatic melanoma. Anti-PD-1 antibodies appear to be well tolerated, and toxicity is manageable. Nivolumab combined with ipilimumab achieves a 5 year survival rate of more than $50 \%$ but at a cost of high toxicity. Ongoing clinical trials investigate novel immunotherapy combinations and strategies (e.g., Talimogene laherparepvec (T-VEC), Bempegaldesleukin (BEMPEG), incorporation or sequencing of targeted therapy, incorporation or sequencing of radiotherapy), and focus on poor prognosis groups (e.g., high tumor burden/LDH levels, anti-PD-1 refractory melanoma, and brain metastases).
\end{abstract}

Keywords: melanoma; PD-1; PD-L1; novel combinations; combination therapies; BEMPEG; T-VEC; LAG-3; IDO1; uveal mealnoma; mucosal melanoma; acral melanoma; desmoplastic melanoma; brain metastases; adjuvant therapy; neoadjuvant therapy

\section{Introduction}

Until recently, distant metastatic melanoma was considered refractory to systemic therapy. Patients with metastatic melanoma had a median overall survival of 6-10 months. A better understanding of the genetic alterations in melanoma cells and the interactions between tumors and the immune system led to the development of immune checkpoint inhibitors (CPIs). The United States Food and Drug Administration (FDA) approval of the anti-cytotoxic T-lymphocyte-associated protein 4 (CTLA-4) antibody ipilimumab in 2011 and the anti-programmed cell death protein 1 (PD-1) antibodies nivolumab and pembrolizumab in 2014 has radically changed the systemic treatment of metastatic 
melanoma and significantly improved its clinical outcome. The second breakthrough in the systemic therapy of metastatic melanoma was the targeted therapy with BRAF and MEK inhibitors that may be used if tumor cells harbor a BRAF-V600 mutation. This review summarizes the current novel data on the treatment of metastatic melanoma with anti-PD-1 antibodies and combinations with other treatment modalities, including clinical trials presented at major conference meetings.

\section{Cutaneous Melanoma}

\subsection{Anti-PD-1 Antibodies}

The PD-1 receptor inhibits T-cell activity by interacting with its ligands: PD-L1 on tumor cells and antigen presenting cells, and PD-L2 on tumor cells, activated monocytes and dendritic cells (DC). This leads to an immunosuppressive tumor environment. The therapeutic use of blocking anti-PD-1 antibodies or anti-PD-L1 antibodies interferes with these immunosuppressive effects and strengthens the T-cell response to the tumor [1] (Figure 1).

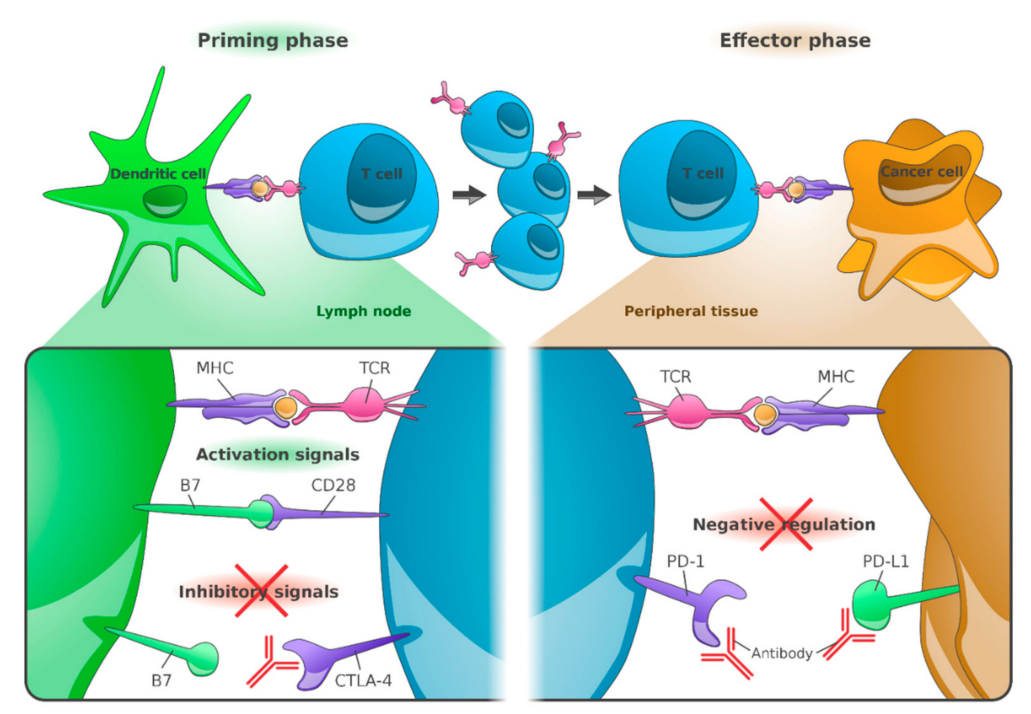

Figure 1. Anti-cytotoxic T-lymphocyte-associated protein 4 (CTLA-4), anti-programmed cell death protein 1 (PD-1) and anti-programmed death ligand 1 (PD-L1) antibodies: Mode of action. Peptides derived from tumor-associated antigens (TAA) are presented by the major histocompatibility complex (MHC) on the surface of cancer cells or DCs and recognized by T cells via their T-cell receptor (TCR). The co-stimulatory molecules B7-1 (or CD80) and B7-2 (or CD86), which are required for T-cell priming, provide an additional signal. T-cell activation leads to upregulation of CTLA-4 on T cells. Binding of CTLA-4 to B7 receptors of dendritic cells results in inhibition of T-cell activation. Anti-CTLA-4 blocking antibodies restore T-cell stimulation in the lymph nodes. Following long-term stimulation, the PD-1 receptor is upregulated by T cells. PD-L1 expressed on cancer cells binds to PD-1 receptors on T cells, which leads to their inhibition. PD-1/PD-L1 antibodies enhance the functional properties of effector T cells at the tumor site. (Reference: Figure adapted from Ribas A [1] and created by F.F. Gellrich).

Nivolumab was the first anti-PD-1 antibody approved for the treatment of melanoma by the FDA in 2014. The approval was based on the results of the phase III study CheckMate 066 [2]. In total, 418 previously untreated patients with metastatic melanoma and wild-type BRAF received either nivolumab or dacarbazine. Nivolumab compared with dacarbazine achieved objective response rates of $40 \%$ vs. $13.9 \%$, median progression-free survival rates of 5.1 months vs. 2.2 months, and 1 year survival rates of $72.9 \%$ vs. $42.1 \%$. National Cancer Institute common terminology criteria for adverse events (CTCAE) grade 3 or 4 drug-related adverse events occurred in $11.7 \%$ of patients treated with nivolumab. An update of the phase I study on nivolumab in patients with metastatic melanoma showed a 5 year survival rate of $34 \%$ [3] (Table 1). 
Table 1. Clinical Studies-Summary.

\begin{tabular}{|c|c|c|c|c|c|c|c|}
\hline Clinical Trial & Phase & Status (January 2020) & Study Treatment & Number of Patients & ORR & OS & References \\
\hline NCT00730639 & 1 & Active, not recruiting & Nivolumab & 418 & $40 \%$ & $34 \%$ ( 5 year) & {$[2,3]$} \\
\hline NCT01295827 & 1 & Completed & Pembrolizumab & 655 & $52 \%$ & $34 \%$ (5 year) & {$[4,5]$} \\
\hline NCT01844505 & 3 & Active, not recruiting & Nivolumab + Ipilimumab & 945 & $58 \%$ & $52 \%$ (5 year) & [6] \\
\hline NCT02089685 & $1 / 2$ & Active, not recruiting & Pembrolizumab + Ipilimumab & 153 & $61 \%$ & $89 \%$ (1 year) & [7] \\
\hline NCT02967692 & 3 & Recruiting & $\begin{array}{c}\text { Spartalizumab }+ \\
\text { Dabrafenib + Trametinib }\end{array}$ & 34 & $75 \%$ & $86 \%$ (1 year) & [8] \\
\hline NCT02130466 & $1 / 2$ & Active, not recruiting & Pembrolizumab + Dabrafenib + Trametinib & 120 & $63 \%$ & $79.9 \%$ (1 year) & [9] \\
\hline NCT02983045 & $1 / 2$ & Recruiting & Nivolumab + BEMPEG (pegIL-2) & 38 & $53 \%$ & N/A & [10] \\
\hline NCT02263508 & 3 & Active, not recruiting & Pembrolizumab + T-VEC & 21 & $67 \%$ & $71 \%$ (3 year) & {$[11,12]$} \\
\hline NCT01968109 & $1 / 2$ & Recruiting & Nivolumab + BMS-986016 (anti-LAG-3) & 43 anti-PD-1 refractory & $16 \%$ & $\mathrm{~N} / \mathrm{A}$ & [13] \\
\hline NCT02752074 & 3 & Completed & Pembrolizumab + epacadostat (IDO1i) & 706 & $34 \%$ & $74 \%$ (1 year)not sig. & [14] \\
\hline
\end{tabular}

BEMPEG: Bempegaldesleukin; pegIL-2: pegylated interleukin 2; T-VEC: Talimogene laherparepvec; LAG-3: Lymphocyte activation gene-3; IDO1i: indoleamine 2,3-dioxygenase-1 inhibitor; ORR: overall response rate; OS: overall survival. 
Pembrolizumab is another anti-PD-1 antibody approved by the FDA in 2014. The studies conducted to date showed comparable results in terms of both efficacy and toxicity [4,15]. KEYNOTE-001 enrolled 655 patients with melanoma. Previously treated or treatment-naive patients with metastatic melanoma received pembrolizumab $2 \mathrm{mg} / \mathrm{kg}$ every 3 weeks, $10 \mathrm{mg} / \mathrm{kg}$ every 3 weeks, or $10 \mathrm{mg} / \mathrm{kg}$ every 2 weeks. The overall response rate (ORR) was $52 \%$ with $25 \%$ complete remissions (CR), and the disease control rate (DCR) was $72 \%$. The median PFS was 8.3 months in all patients and 16.9 months in treatment-naive patients, and the estimated 5 year overall survival (OS) rate was $34 \%$ and $41 \%$, respectively. Treatment-related adverse events (TRAEs) occurred in $86 \%$ of patients, including $17 \%$ CTCAE grade 3 or 4 TRAEs. TRAEs led to study discontinuation in $7.8 \%$ of patients [5] (Table 1 ).

In conclusion, the anti-PD-1 antibodies nivolumab and pembrolizumab achieved an ORR of $40 \%$ to $50 \%$ and 5 year OS rates of 30 to $40 \%$ in patients with metastatic melanoma. Responses are durable. Toxicity is manageable. Most adverse events are immune-mediated adverse reactions such as endocrinopathies, pneumonitis, colitis, nephritis, and hepatitis [16].

\subsection{Combination of Anti-PD-1 Antibodies with Anti-CTLA-4 Antibodies}

The combined blockade of PD-1 and CTLA-4 enables inactivated tumor-specific T cells to multiply again and perform their effector function. This leads to immune activation of the so far immunosuppressed tumor microenvironment [17] (Figure 1).

In the phase III CheckMate 067 trial [6], 945 therapy-naive patients with metastatic melanoma received the anti-PD-1 antibody nivolumab in combination with the anti-CTLA-4 antibody ipilimumab or nivolumab, or ipilimumab as monotherapy. This study was insufficiently powered in terms of a reliable comparative statistical evaluation of nivolumab plus ipilimumab vs. nivolumab. Objective response rates were $58 \%$ for the combination vs. $45 \%$ for nivolumab vs. $19 \%$ for ipilimumab. The combination of nivolumab with ipilimumab achieved a 5 year survival rate of $52 \%$ vs. $44 \%$ for nivolumab monotherapy vs. $26 \%$ for ipilimumab monotherapy. The most common adverse events were colitis and hepatitis under treatment with nivolumab +/- ipilimumab and exanthema, colitis and hypophysitis under ipilimumab monotherapy. CTCAE grade 3 and 4 adverse events were observed in $59 \%, 23 \%$ and $28 \%$ of the patients in the nivolumab plus ipilimumab, nivolumab, and ipilimumab group, respectively. Most CTCAE grade 3 and 4 adverse events improved under adequate immunosuppressive therapy [18]. The median time to resolution of adverse events was less than 12 weeks, with the exception of some events that had not yet resolved (e.g., endocrine events). No unknown long-term toxicity was reported (Table 1).

In the KEYNOTE-029 study, the anti-PD-1 antibody pembrolizumab was combined with ipilimumab. In total, $72 \%$ of patients received all 4 doses of pembrolizumab $2 \mathrm{mg} / \mathrm{kg}$ plus ipilimumab $1 \mathrm{mg} / \mathrm{kg}$. $42 \%$ of patients remained on pembrolizumab monotherapy. In total, $61 \%$ of patients achieved an objective response. Estimated 1 year PFS was $69 \%$ and estimated 1 year OS was $89 \%$ [7]. In total, $60 \%$ of patients developed therapy-related adverse events and CTCAE grade 3 and 4 adverse events were observed in $27 \%$ of patients. Most common adverse events were hypothyroidism (16\%) and hyperthyroidism (11\%). No treatment-related deaths occurred.

In conclusion, the combination of nivolumab and ipilimumab appears to be superior to anti-PD-1 monotherapy in the treatment of metastatic melanoma. However, higher response rates ( $58 \%$ vs. $44 \%$ ) and better 4 year survival rates (53\% vs. $46 \%$ ) are at the expense of higher toxicity (grade 3 and $4: 55 \%$ vs. $16 \%$ ). The combination of pembrolizumab with low-dose ipilimumab achieved comparable high response rates with lower toxicity. However, this combination is not yet approved.

\subsection{Duration of Therapy}

In the 4 year update of the CheckMate 067 trial, the data of 103 patients who completed 2 years of pembrolizumab were analyzed [19]. The median follow-up after end of treatment was 20.3 months. Complete remission was achieved by $27.2 \%$ of patients, partial remission (PR) by $63.1 \%$ and stable 
disease (SD) by $9.7 \% .18$ months after completion of therapy, and $95.8 \%$ of patients with CR, $91.3 \%$ of patients with PR and $66.7 \%$ of patients with SD were still progression free.

In summary, anti-PD-1 antibodies show a durable antitumor activity in patients who have completed 2 years of therapy. However, further studies are required to determine the optimal therapy duration.

\subsection{Combination of Targeted Therapy with Anti-PD-1 Antibodies}

Targeted therapy, i.e., BRAF inhibitors combined with MEK inhibitors, induce rapid responses and high response rates in patients with metastatic melanoma in the presence of a BRAF-V600 mutation [20]. However, in the majority of patients, the duration of response is limited due to acquired resistance [21]. By contrast, CPIs are slower-acting and induce less frequent but durable responses. There is growing evidence that BRAF/MEK inhibitors target multiple events in the cancer-immunity cycle. For example BRAF/MEK inhibitors promote the release of cancer cell antigens, cancer antigen presentation, infiltration of $\mathrm{T}$ cells into tumors, recognition of cancer cells by $\mathrm{T}$ cells and killing of cancer cells [22]. Combining targeted with immune therapy may achieve rapid responses, high response rates and durable responses with prolonged survival.

A phase III study (NCT02967692) is investigating the safety and efficacy of the anti-PD-1 antibody spartalizumab in combination with the BRAF inhibitor dabrafenib and the MEK inhibitor trametinib in untreated patients with BRAF V600-mutant metastatic melanoma. The data of 36 patients enrolled in part 1 (safety run-in cohort) and part 2 (biomarker cohort) of this study were presented at the 2019 ASCO Annual Meeting [8]. Treatment with spartalizumab combined with dabrafenib and trametinib achieved an ORR of $75 \%$ and a complete response rate of $42 \%$. The 1 year OS rate was $86 \%$, and the median OS was not reached. Toxicity appears to be high. CTCAE grade $\geq 3$ adverse events occurred in $78 \%$ of patients, and adverse events leading to discontinuation of all three study drugs occurred in $17 \%$ patients. Adverse events included pyrexia, chills, fatigue, cough, and arthralgia [8]. The global part 3 of this study, which compares spartalizumab in combination with dabrafenib and trametinib with dabrafenib and trametinib alone, is ongoing (Table 1).

In the KEYNOTE-022 phase-2 study (NCT02130466), 120 treatment-naive BRAF-V600E/K-mutant patients with advanced melanoma were randomized to receive the BRAF inhibitor dabrafenib and the MEK inhibitor trametinib in combination with pembrolizumab or placebo [9]. ORR was $63.3 \%$ in the triplet group compared with $71.7 \%$ in the doublet group. The reason for this difference was considered to be an imbalance in baseline patient characteristics with better prognostic factors in the doublet arm. PFS was 16.0 months in the triplet group vs. 10.3 months in the doublet group. However, the planned benefit for a statistically significant improvement was not reached. The 1 year OS rate was $79.9 \%$ and $72.9 \%$, respectively (Table 1). After end of study treatment, a greater proportion of patients in the doublet arm $(48.3 \%)$ received post-progression immunotherapy than in the triplet arm (15.0\%). Grade 3-5 treatment-related adverse events occurred in 58.3\% in the triplet arm and $26.7 \%$ in the doublet arm. The most common adverse events were fever, hepatitis and rash.

In phase 1 of the KEYNOTE-022 trial (NCT02130466), 15 BRAF-V600E/K-mutant patients were enrolled for triplet therapy. ORR was $73 \%$ [23]. Remarkably, $40 \%$ of the patients continued to respond at a median follow-up of 27 months, while the median duration of response was approximately 1 year for doublet therapy with BRAF and MEK inhibitors [24].

In conclusion, the efficacy of the anti-PD-1 antibodies spartalizumab or pembrolizumab combined with the BRAF inhibitor dabrafenib and the MEK inhibitor trametinib may exceed the efficacy of dabrafenib and trametinib alone. Toxicity appears to be high, but manageable.

\subsection{Combination of Anti-PD-1 Antibodies with Pegylated Engineered Interleukin-2}

Bempegaldesleukin (BEMPEG; NKTR-214) is a prodrug of conjugated interleukin (IL)-2. The IL-2 core is conjugated to six releasable polyethylene glycol (PEG) chains. In vivo, the PEG chains slowly release to generate active IL-2 conjugates, selectively stimulating CD8+ T cells over regulatory T cells 
(Figure 2). BEMPEG increases tumor-infiltrating lymphocytes, T-cell clonality and PD-1 expression [25]. In an ongoing phase I/II study, the combination of BEMPEG with the anti-PD-1 antibody nivolumab in patients with previously untreated metastatic melanoma is being investigated. At the 2019 ASCO Annual Meeting, the data of 38 efficacy-evaluable patients were presented [10]. BEMPEG combined with nivolumab achieved an ORR of $53 \%$, a CR in $34 \%$ of patients, and a DCR of $74 \%$. The responses were durable with $80 \%$ ongoing responses after a median follow-up of 12.7 months. Biomarker analyses of baseline tumor biopsies identified immune signatures that accumulate for treatment response, e.g., interferon (IFN) $\gamma$ or CD8+ tumor-infiltrating lymphocytes (TIL). Notably, responses were even observed in patients with the least favorable tumor microenvironment. The most common adverse events were flu-like symptoms, rash and fatigue. Therapy had to be discontinued by $9.8 \%$ of patients due to TRAEs [10] (Table 1).

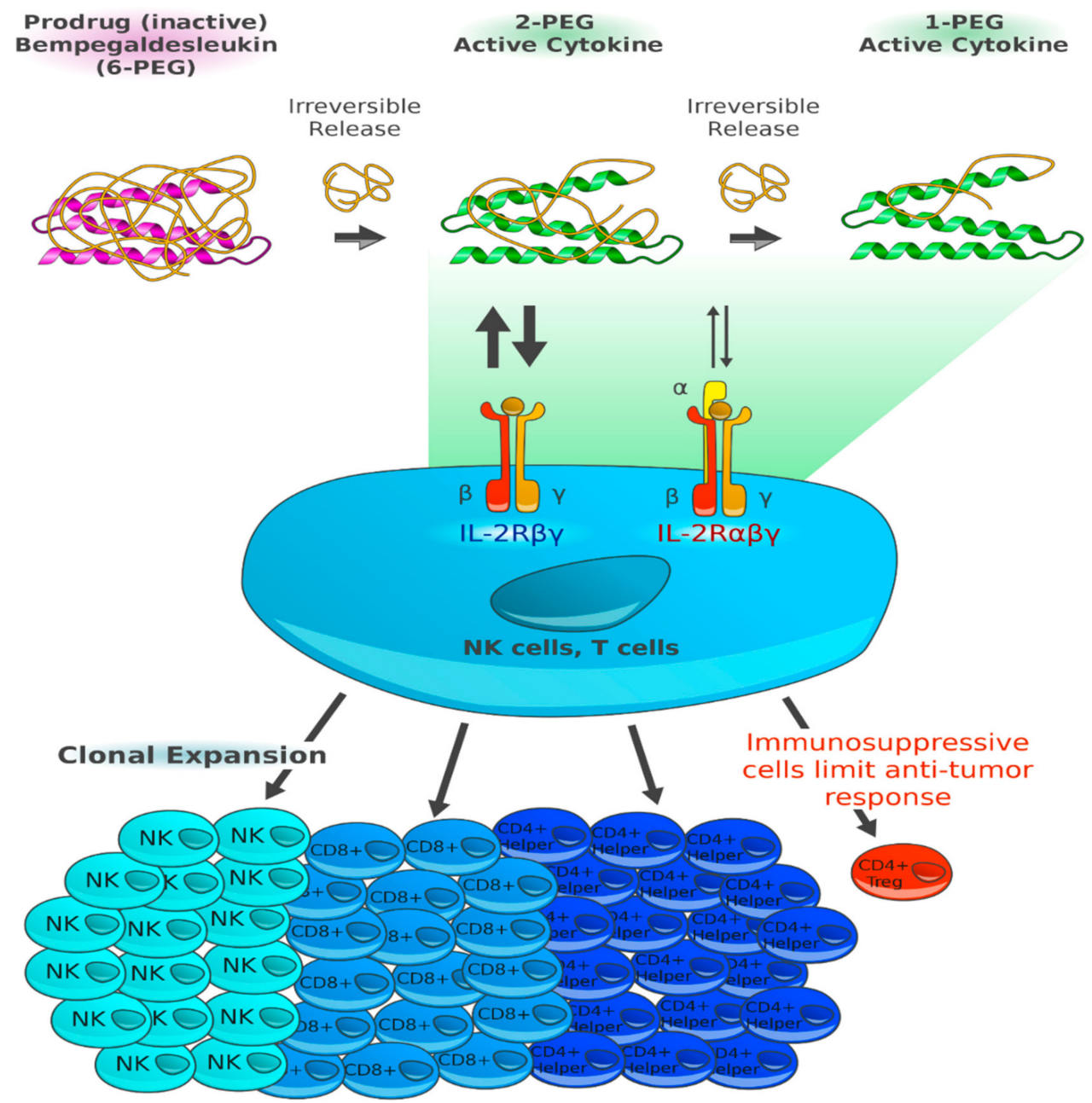

Stimulates Immune Response to Kill Tumor Cells

Figure 2. Bempegaldesleukin expands and activates cluster of differentiation (CD)8+ effector T cells and natural killer cells over regulatory T cells. Bempegaldesleukin (BEMPEG; NKTR-214) is a human recombinant inteleukin-2 (IL-2) attached to six releasable polyethylene glycol (PEG) chains that alter its pharmacokinetics and its receptor binding. When fully PEGylated, NKTR-214 is a prodrug with no biological activity. In vivo, the PEG chains slowly release to generate active IL-2 conjugates with limited binding to the IL-2R $\alpha$ subunit, thereby favoring the dimeric $\beta \gamma$-IL-2 receptor (IL-2R $\beta \gamma$; CD122). Consequently, NKTR-214 is selectively stimulating CD8+ T cells and natural killer (NK) cells over the undesirable T regulatory cells (Treg) [25]. (Reference: Figure adapted from Charych D [26] and created by F.F. Gellrich). 
In conclusion, the biomarker analyses identified baseline immune signatures that correlated with response to BEMPEG combined with nivolumab. Responses were seen in both favorable and unfavorable tumor microenvironments.

\subsection{Combination of Anti-PD-1 Antibodies with T-VEC}

Talimogene laherparepvec (T-VEC) is the first oncolytic virus therapy approved for patients with metastatic melanoma. T-VEC is a type 1 herpes simplex virus genetically modified to preferentially replicate in tumor cells. In tumor cells, the virus replicates and secretes Granulocyte-macrophage colony-stimulating factor (GM-CSF). The tumor cells lyse and release viruses, GM-CSF and tumor cell-associated antigens. GM-CSF attracts DCs that process and present tumor cell antigens to T cells. $\mathrm{T}$ cells are then programmed to identify and kill tumor cells [27] (Figure 3). Altogether, T-VEC creates an immunogenic tumor microenvironment and may enhance the efficacy of anti-PD-1 antibodies. In particular non-immunogenic tumors may benefit from a combination of T-VEC with CPIs [28].

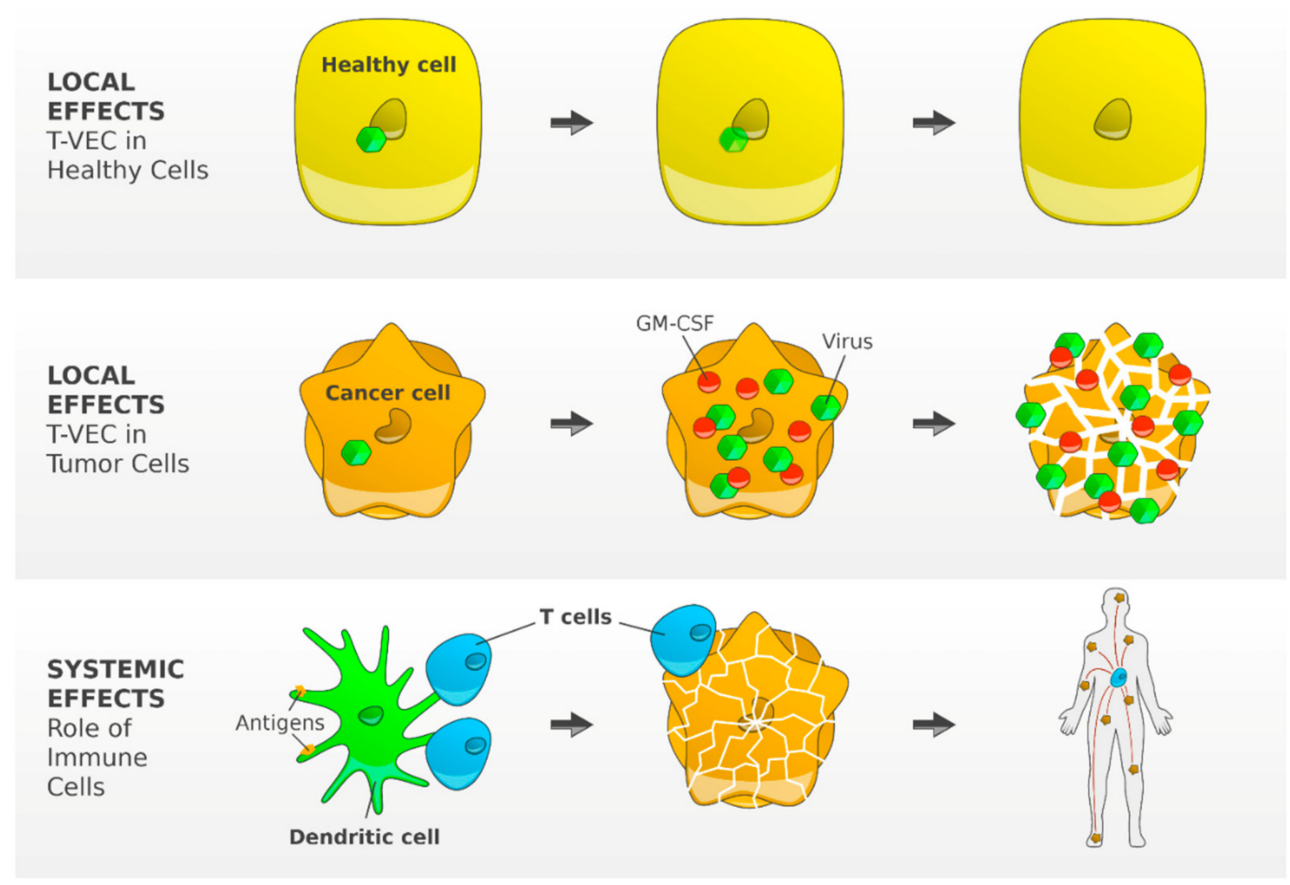

Figure 3. T-VEC - mechanism of action. T-VEC is a type 1 herpes simplex virus genetically modified by the deletion of two non-essential viral genes. The functional deletion of the gene herpesvirus neurovirulence factor (ICP34.5) attenuates viral pathogenicity and enhances tumor-selective replication. Inside a normal cell, the virus is unable to replicate. In tumor cells, the virus replicates and induces granulocyte-macrophage colony-stimulating factor (GM-CSF). The lysed tumor cells release viruses, GM-CSF and TAAs. GM-CSF attracts DCs that process and present TAAs to T cells resulting in an efficient induction and activation of tumor-reactive T cells [27]. (Reference: Figure adapted from Andtbacka R [27] and created by F.F. Gellrich).

In a phase Ib study, patients with metastatic melanoma and injectable lesions without prior systemic therapy were treated with T-VEC intralesionally and the anti-PD-1 antibody pembrolizumab. In total, 21 patients were enrolled, and the maximum treatment period was 2 years. At the 2018 Society for Melanoma Research Annual Meeting, the updated data of this study were presented. The median follow-up time was 36.8 months. Therapy was generally well tolerated, with fatigue, fever, and chills as the most common adverse events. The ORR was $67 \%$ with a complete response rate of $43 \%$ and $57 \%$ of patients remained in response [11,12]. Median progression-free survival (PFS) and OS were not reached at the data cutoff. The 36 month PFS and OS rates were $53.6 \%$ and $71 \%$, respectively (Table 1 ). 
In this study, the combination of T-VEC and pembrolizumab was well tolerated and induced durable responses in the majority of patients. The randomized phase III study comparing T-VEC combined with pembrolizumab to pembrolizumab alone (NCT02263508) has completed enrollment and is currently ongoing [29].

\subsection{Combination of Anti-PD-1 Antibodies with Anti-Lymphocyte Activation Gene (LAG)-3 Antibodies}

Lymphocyte activation gene-3 (LAG-3) is an immune cell receptor that regulates a checkpoint pathway limiting the activity of T cells. Signaling via LAG-3 and other T-cell inhibitory receptors (e.g., PD-1) can lead to T-cell dysfunction and tumor immune escape. Simultaneous blockade of LAG-3 and PD-1 may synergistically restore T-cell activation and increase antitumor immunity [13].

In a phase 1/2a study (NCT01968109), the anti-LAG-3 antibody BMS-986016 in combination with the anti-PD-1 antibody nivolumab demonstrated peripheral T-cell activation, preliminary clinical activity and tolerability [30]. Patients progressed on prior anti-PD-1/PD-L1 therapy were treated with anti-LAG-3 antibody in combination with nivolumab. ORR was $16 \%$ and DCR was $45 \%$. Grade $3 / 4$ treatment-related adverse events occurred in $9 \%$ of patients [13] (Table 1).

In conclusion, the anti-LAG-3 antibody BMS-986016 showed efficacy in anti-PD-1/PD-L1-refractory patients, while toxicity is comparable to nivolumab monotherapy.

\subsection{Combination of Anti-PD-1 Antibodies with IDO1 Inhibitors}

Tumors may evade immunosurveillance through upregulation of the enzyme indoleamine 2,3-dioxygenase 1 (IDO1). In the phase 1/2 ECHO-202/KEYNOTE-037 study, the IDO1 inhibitor epacadostat in combination with the anti-PD-1 antibody pembrolizumab was well tolerated and showed encouraging antitumor activity in multiple advanced tumors [31]. Based on this trial, 706 patients with unresectable stage III or IV melanoma were randomly assigned to receive pembrolizumab plus epacadostat or placebo [14]. In this phase 3 study (NCT02752074), epacadostat plus pembrolizumab did not improve PFS or OS compared with placebo plus pembrolizumab (Table 1).

\section{Adjuvant Therapy}

There is a substantial risk of metastasis in resected high-risk melanoma. In patients with resected locoregional metastases, 1 year recurrence rates range from $8 \%$ to $76 \%$ and 10 year melanoma-specific survival rates range from $24 \%$ to $88 \%$, depending on the tumor load [32-34]. For many years, no adjuvant therapy was available to be effective in reducing recurrence and mortality in resected high-risk melanoma. Recent developments in adjuvant therapy have changed the standard of care for these patients.

In 2015, the FDA approved the anti-CTLA-4 antibody ipilimumab as an adjuvant therapy for patients with melanoma with regional lymph node metastases who have undergone complete resection. In a phase III trial, 951 patients were randomly assigned to ipilimumab $10 \mathrm{mg} / \mathrm{kg}$ or placebo every 3 weeks for four doses, then every 3 months for up to 3 years. The rate of OS at 5 years was $65.4 \%$ in the ipilimumab group, as compared with $54.4 \%$ in the placebo group. However, toxicity was high. CTCAE grade 3 or 4 immune-related adverse events occurred in $41.6 \%$ of patients in the ipilimumab group and in $2.7 \%$ of patients in the placebo group. In the ipilimumab group, 5 patients $(1.1 \%)$ died due to immune-related adverse events [35,36].

In the phase III CheckMate 238 trial [37], patients who had undergone complete resection of locoregional or distant metastases were treated with anti-PD-1 nivolumab or anti-CTLA-4 ipilimumab for one year. The 1 year recurrence-free survival rates were $70.5 \%$ in the nivolumab arm and $60.8 \%$ in the ipilimumab arm. At a minimum follow-up of 24 months, recurrence-free survival rates continued to be higher for nivolumab vs. ipilimumab with $62.6 \%$ vs. $50.2 \%$ [38]. Toxicity was lower in the nivolumab arm with adverse events requiring therapy discontinuation in $9.7 \%$ of patients compared with $42.6 \%$ of patients in the ipilimumab arm. Altogether, adjuvant therapy with nivolumab achieved an improved recurrence-free survival and a lower rate of toxicity, compared with ipilimumab. 
In the phase III EORTC 1325 study [39], patients with completely resected locoregional metastases received anti-PD-1 pembrolizumab (514 patients) or placebo (505 patients) for 1 year. At a median follow-up of 15 months, pembrolizumab was associated with significantly longer recurrence-free survival than placebo with a 1 year rate of recurrence-free survival of $75.4 \%$ vs. $61.0 \%$. CTCAE grade $3-5$ adverse events were reported in $14.7 \%$ of the patients in the pembrolizumab group and in $3.4 \%$ of patients in the placebo group. There was one treatment-related death due to myositis in the pembrolizumab group.

In summary, adjuvant CPI therapy with anti-CTLA-4 ipilimumab showed both a recurrence-free survival and overall survival benefit, but at a cost of high toxicity. Anti-PD-1 antibodies such as nivolumab and pembrolizumab, achieved higher recurrence-free survival rates with lower toxicity. Given the significant recurrence-free survival benefit, a significant overall survival benefit is expected. Nivolumab was approved for adjuvant treatment of patients who had undergone resection of melanoma and resection of all sites of disease. Pembrolizumab was approved for adjuvant treatment of patients who have melanoma with lymph node involvement who underwent complete resection.

\section{Neoadjuvant Therapy}

Besides adjuvant treatment, patients with regional lymph node metastases may also be treated in the neoadjuvant setting, before undergoing resection. Advantages may be the induction of a stronger immune response and the reduction of tumor burden. Treatment response may provide a predictive marker for additional adjuvant therapy.

In a phase II trial investigating different neoadjuvant strategies, 86 patients were included [40]. In arm A, patients received $2 \times$ ipilimumab $3 \mathrm{mg} / \mathrm{kg}$ plus nivolumab $1 \mathrm{mg} / \mathrm{kg}$; in arm B, $2 \times$ ipilimumab $1 \mathrm{mg} / \mathrm{kg}$ plus nivolumab $3 \mathrm{mg} / \mathrm{kg}$; and in arm C, $2 \times$ ipilimumab $3 \mathrm{mg} / \mathrm{kg}$ followed immediately by $2 \times$ nivolumab $3 \mathrm{mg} / \mathrm{kg}$. CTCAE grade $\geq 3$ AEs occurred in $40 \%, 20 \%$, and $50 \%$ in arm A, B and C, respectively. Arm $C$ was closed due to high toxicity. Pathologic response rates were $80 \%$ and $77 \%$ in arm $\mathrm{A}$ and B with $43 \%$ and $57 \%$ complete responses, respectively. Pathologic response appeared to be the strongest marker for relapse-free survival. Altogether, ipilimumab $1 \mathrm{mg} / \mathrm{kg}$ plus nivolumab $3 \mathrm{mg} / \mathrm{kg}$ appeared to be the most attractive for further studies.

In summary, CPIs have also been investigated in patients with palpable lymph node metastases in the neoadjuvant setting. These studies show promising results and further studies are expected in the near future.

\section{Brain Metastases}

Melanoma, along with lung and breast cancer, is one of the most common causes of brain metastases. The prognosis for melanoma patients with brain metastases is unfavorable with a median overall survival of approximately 4 months. The established local treatments include surgical resection, stereotactic radiosurgery and whole brain radiotherapy [41].

The approval of effective targeted and immune therapies has significantly improved the prognosis of metastatic melanoma including brain metastases with a median OS for patients with brain metastases of approximately 7 months for anti-CTLA-4 ipilimumab [42], 10 months for anti-PD-1 pembrolizumab or nivolumab [43] and up to 24 months for BRAF and MEK inhibitors [44]. Recently, combination therapy with anti-PD1 and anti-CTLA-4 showed unprecedented results $[43,45]$.

\subsection{Nivolumab Plus Ipilimumab}

At the 2019 ASCO Annual Meeting, updated efficacy and safety results of the phase II study CheckMate 204 (NCT02320058) for patients with melanoma brain metastases (MBM) were reported [46]. In this phase II study, patients with MBM were enrolled into two cohorts: (cohort A) patients with asymptomatic brain metastases and no steroid treatment, and (cohort B) patients with symptomatic brain metastases with or without steroid treatment. All patients received nivolumab plus ipilimumab (4 times), then nivolumab until tumor progression or toxicity. In cohort A, the updated data of 101 
asymptomatic MBM patients with a median follow-up of 20.6 months were reported. Intracranial and extracranial responses were largely concordant with an intracranial objective response rate of $54 \%$. In total, $87 \%$ of patients showed an ongoing response. The overall survival rate at 18 months was $75 \%$, the median OS has not been reached. In cohort B, new data of 20 patients with a median follow-up of 5.2 months were presented. The intracranial objective response rate dropped to $16.7 \%$, and the median overall survival was 8.7 months. In both cohorts, more than $50 \%$ of patients experienced grade 3 to 4 adverse events that were comparable to patients without brain metastases [47].

In the ABC trial (NCT02374242), 76 patients with MBM were enrolled in three cohorts. Patients with asymptomatic MBM without prior local brain therapy were treated with nivolumab plus ipilimumab (cohort A) or nivolumab (cohort B). Previously treated patients, patients with neurological symptoms or leptomeningeal metastases were treated with nivolumab (cohort $\mathrm{C}$ ). The 3 year overall survival rates were $49 \%$ (cohort A) vs. $42 \%$ (cohort B) vs. $19 \%$ (cohort C). Intracranial response rates were $51 \%$ (cohort A) vs. $20 \%$ (cohort B) vs. $6 \%$ (cohort C). Grade 3 or 4 treatment-related adverse events occurred in $54 \%$ patients of cohort $A, 20 \%$ in cohort B and $13 \%$ in cohort C $[43,48]$.

\subsection{Radiotherapy Combined with Immune Checkpoint Inhibitors}

Despite the encouraging study data, approximately half of patients with MBM fail to respond to systemic therapy. Response duration appears to be shorter than that in extracranial disease, emphasizing the need of combination or new treatment strategies. Several retrospective analyses suggest that combining stereotactic radiosurgery (SRS) with active systemic therapies improves melanoma brain metastases (MBM) control and prolongs survival without increasing toxicity [44,49]. Preclinical and clinical data suggest that radiotherapy can cause disruption of the blood-brain barrier enhancing drug delivery to the brain [50]. Moreover, the combination of radiotherapy and immune CPIs may increase the antitumor response by promoting antigen presentation and T-cell activation [51,52].

In a large real-life cohort of patients with MBM treated with CPIs or BRAF/MEK inhibitors, the risk of death was decreased by $40 \%$ for patients treated with radiotherapy, in comparison with those who did not receive radiotherapy [44]. In a retrospective study, data of 208 patients treated with SRS or whole brain radiation therapy in combination with CPIs or BRAF/MEK inhibitors within a 6-week interval to radiotherapy were analyzed [49]. The best survival was seen in patients treated with anti-PD-1 plus anti-CTLA-4 or anti-PD-1 alone combined with SRS with 12 month survival rates of $100 \%$ and $70 \%$, respectively.

\subsection{Immune Checkpoint Inhibitors in Melanoma Brain Metastases—Conclusions}

In patients with asymptomatic MBM, the anti-PD-1 antibody nivolumab combined with the anti-CTLA-4 antibody ipilimumab achieved a high rate of durable intracranial responses supporting nivolumab plus ipilimumab as a first-line treatment in these patients. Patients with symptomatic MBM are challenging to treat. Some patients can benefit from nivolumab plus ipilimumab. Further studies in patients with symptomatic MBM are required to evaluate combination or new treatment strategies such as radiotherapy combined with CPIs or combinations of BRAF/MEK inhibitors with CPIs. Retrospective studies suggest that combining stereotactic radiosurgery with CPIs prolongs overall survival.

\section{Uveal Melanoma}

Uveal melanoma (UM) is rare and accounts for approximately $5 \%$ of melanoma cases [53]. UM is characterized by mutations in GNAQ or GNA11 resulting in activation of the mitogen-activated protein kinase (MAPK) and other signaling pathways [54,55]. Approximately $40 \%-50 \%$ of patients with UM will develop metastatic disease. Metastatic UM is often fatal because the most common metastatic site is the liver [56]. There is currently no standard therapy for metastatic UM. Treatment options proposed in patients with metastatic UM are the same as those in patients with cutaneous 
melanoma. The prognosis of uveal melanoma is poor compared to cutaneous melanoma posing a particular treatment challenge.

In a French retrospective study [57], 210 patients with metastatic UM were treated with the anti-CTLA-4 antibody ipilimumab or the anti-PD-1 antibodies nivolumab or pembrolizumab. No partial or complete response of metastases was observed. SD was seen in $32 \%$ of patients. OS of patients treated with immunotherapy was not significantly different from those of patients treated with chemotherapy, with a median OS of 13.38 months vs. 11.02 months.

In a German retrospective study [58], 86 patients with metastatic UM were treated with anti-PD-1 antibodies with a response rate of $4.7 \%$ and a median OS of 14 months for pembrolizumab-treated and 10 months for nivolumab-treated patients. Fifteen patients were treated with anti-PD-1 antibodies combined with anti-CTLA-4 antibodies with a PR seen in two patients and a median OS not reached.

In a phase II study, 35 patients with previously untreated or treated metastatic UM received anti-PD-1 nivolumab combined with anti-CTLA-4 ipilimumab [59]. A PR was seen in $17 \%$ of patients, and a stable disease in $53 \%$ of patients. Median OS was 1.6 years, and the 1 year OS rate was $62 \%$. CTCAE grade 3 or 4 adverse events occurred in $40 \%$ of patients, $29 \%$ of patients had to discontinue therapy.

In summary, single-agent CPI therapy in metastatic uveal melanoma has yielded poor results. Anti-PD-1 combined with anti-CTLA-4 has the potential to improve response rates and survival. However, toxicity is high.

\section{Mucosal Melanoma}

Mucosal melanoma is a rare subtype of melanoma, and occurs in the sinonasal cavity, oral cavity, anorectal region, or urogenital tract $[60,61]$. The prognosis of mucosal melanoma appears to be poorer than that of cutaneous melanoma, since mucosal melanoma is often diagnosed at an advanced stage and has particular clinical and genetic characteristics [62].

In a French multicenter retrospective study, 151 patients with metastatic mucosal melanoma received immunotherapy with anti-CTLA-4 (50.3\%) or anti-PD-1 antibodies $(49.7 \%)$. The objective response rate was $11.9 \%$, and the DCR was $29.8 \%$. The OS of mucosal melanoma patients treated with CPIs appeared to be longer than that of patients treated with chemotherapy, with a median OS of 15.97 months and 8.82 months, respectively. In conclusion, immunotherapy appears to improve overall survival for patients with metastatic mucosal melanoma [57]. In a pooled analysis [63], the outcome of patients with metastatic mucosal melanoma treated in trials either with single-agent nivolumab $(n=86)$ or the combination of nivolumab and ipilimumab $(n=35)$ was reported. For nivolumab monotherapy, the objective response rate was $23.3 \%$ in patients with mucosal melanoma, compared with $40.9 \%$ for patients with cutaneous melanoma. Median PFS was 3.0 months and 6.2 months for mucosal and cutaneous melanoma. Treatment with the combination of nivolumab and ipilimumab achieved an ORR of $37.1 \%$ in mucosal melanoma, compared with $60.4 \%$ seen in patients with cutaneous melanoma. Median PFS was 5.9 months and 11.7 months for mucosal and cutaneous melanoma. CTCAE grade 3 or 4 adverse events occurred in $8.1 \%$ of patients under treatment with nivolumab and in $40.0 \%$ of patients under treatment with nivolumab plus ipilimumab.

\section{Mucosal Melanoma-Conclusions}

Immunotherapy can be successful in mucosal melanoma. However, response rates are lower than in cutaneous melanoma. Anti-PD-1 nivolumab combined with anti-CTLA-4 ipilimumab appears to have greater efficacy than nivolumab alone. Clinical trials for patients with mucosal melanoma remain a key priority.

\section{Acral and Desmoplastic Melanoma}

Acral lentiginous melanomas are considered a distinct subgroup with unique clinical, morphologic, and genetic characteristics [62], lower tumor mutational burden [64] and poorer prognosis than 
non-acral cutaneous melanomas [65]. In a retrospective analysis, 25 patients with acral melanoma received pembrolizumab or nivolumab [66]. ORR was 32\%, median PFS 4.1 months and median OS 31.7 months, supporting the use of PD-1 blockade in clinical practice.

Desmoplastic melanoma is characterized by a lack of actionable driver mutations and is highly associated with UV-induced DNA damage [67]. In a retrospective study, 60 patients with advanced desmoplastic melanoma treated with anti-PD-1 or anti-PD-L1 antibodies were identified [68]. ORR was $70 \%$ with $32 \%$ complete remissions. Patients with advanced desmoplastic melanoma appear to benefit from anti-PD-1/PD-L1 therapy. The benefit is expected to result from the high mutation burden.

\section{Immune-Related Adverse Events}

Therapy with CPIs is associated with a wide spectrum of adverse events related to the mechanism of action. ICIs can induce immune-related adverse events (irAEs) in all organ systems, and most commonly affect the skin, gastrointestinal tract, lungs, and the endocrine, musculoskeletal, renal, nervous, hematologic, cardiovascular, and ocular systems. Severe irAEs occur in 10 to $20 \%$ of patients under monotherapy with nivolumab or pembrolizumab [2,5] and in more than $50 \%$ of patients under nivolumab combined with ipilimumab [69]. IrAEs may affect quality of life, may cause loss of organ function, and may even lead to death. Hence, toxicity of ICIs requires early detection and competent management, and patients and physicians should be aware that any symptoms may be treatment-related. The ASCO has developed guidelines on the management of irAEs [70]. General recommendations include: (1) Other causes should be excluded (e.g., infection, tumor progression). (2) For grade 2 toxicities corticosteroids may be administered. (3) For grade 3 toxicities, high-dose corticosteroids may be administered and subsequently tapered for at least 4 weeks. (4) If there is no improvement within 48 to $72 \mathrm{~h}$, immunosuppressive therapy may be escalated (e.g., infliximab).

Notably, it was recently shown that treatment of mice with tumor necrosis factor (TNF) inhibitors concomitantly with anti-PD-1 and anti-CTLA-4 antibodies ameliorates immune-related colitis and, in addition, improves anti-tumor efficacy [71]. These data suggest that it is feasible to dissociate efficacy and toxicity of combined immune checkpoint blockade.

\section{Conclusions}

Impressive progress has been made in the treatment of patients with metastatic melanoma. Immune checkpoint inhibitors, in particular anti-PD-1 antibodies such as pembrolizumab and nivolumab and the combination of nivolumab with the anti-CTLA-4 antibody ipilimumab, can achieve long-term survival for patients with metastatic melanoma with 5 year survival rates of more than $40 \%$ and $50 \%$, respectively. The anti-PD-1 antibodies nivolumab and pembrolizumab were also approved for adjuvant treatment of patients with resected metastatic melanoma.

Anti-PD-1 antibodies appear to be well tolerated, and toxicity is manageable. However, immune-related adverse events may be irreversible in rare cases. Patients treated with nivolumab combined with ipilimumab achieve a 5 year survival rate of $52 \%$ but at a cost of high toxicity with very rare cases of fatal outcome.

However, there are patients who do not benefit from treatment. These poor prognosis groups include patients with high tumor burden or high lactate dehydrogenase (LDH) levels, uveal melanoma, mucosal melanoma, brain metastases and anti-PD-1 refractory melanoma.

Ongoing clinical trials investigate novel immunotherapy combinations and strategies (e.g., T-VEC, BEMPEG, incorporation or sequencing of targeted therapy, and incorporation or sequencing of radiotherapy), and focus on poor prognosis groups (e.g., high tumor burden/LDH levels, anti-PD-1 refractory melanoma, and brain metastases).

Author Contributions: Conceptualization, F.F.G. and F.M.; methodology, F.F.G. and F.M.; validation, M.S., S.B. and F.M.; resources, S.B.; writing—original draft preparation, F.F.G. and F.M.; writing —review and editing, F.F.G., M.S., S.B. and F.M.; visualization, F.F.G.; supervision, M.S.; project administration, M.S.; funding acquisition, M.S. All authors have read and agreed to the published version of the manuscript. 
Funding: Open Access Funding by the Publication Fund of the TU Dresden

Conflicts of Interest: The authors declare no conflict of interest.

\section{Abbreviations}

FDA United States Food and Drug Administration

CTLA-4 cytotoxic T-lymphocyte-associated protein 4

PD-1 programmed cell death protein 1

ASCO American Society of Clinical Oncology

CTCAE criteria for adverse events

ORR overall response rate

CR complete remission

PR partial remission

SD stable disease

DCR disease control rate

OS overall survival

PFS progression-free survival

TRAE treatment related adverse event

irAE immune-related adverse event

CPI immune checkpoint inhibitor

IL-2 interleukin-2

PEG polyethylene glycol

T-VEC talimogene laherparepvec

GM-CSF granulocyte-macrophage colony-stimulating factor

MBM melanoma brain metastases

UM uveal melanoma

MAPK mitogen-activated protein kinase

MHC major histocompatibility complex

TCR T-cell receptor

TAA tumor-associated antigens

DC dendritic cell

NK natural killer cells

Treg T regulatory cells

\section{References}

1. Ribas, A. Tumor immunotherapy directed at PD-1. N. Engl. J. Med. 2012, 366, 2517-2519. [CrossRef] [PubMed]

2. Robert, C.; Long, G.V.; Brady, B.; Dutriaux, C.; Maio, M.; Mortier, L.; Hassel, J.C.; Rutkowski, P.; McNeil, C.; Kalinka-Warzocha, E.; et al. Nivolumab in previously untreated melanoma without BRAF mutation. N. Engl. J. Med. 2015, 372, 320-330. [CrossRef] [PubMed]

3. Hodi, F.S.; Kluger, H.; Sznol, M.; Carvajal, R.; Lawrence, D.; Atkins, M.; Powderly, J.; Sharfman, W.; Puzanov, I.; Smith, D.; et al. Abstract CT001: Durable, long-term survival in previously treated patients with advanced melanoma (MEL) who received nivolumab (NIVO) monotherapy in a phase I trial. Cancer Res. 2016, 76, CT001.

4. Robert, C.; Ribas, A.; Wolchok, J.D.; Hodi, F.S.; Hamid, O.; Kefford, R.; Weber, J.S.; Joshua, A.M.; Hwu, W.J.; Gangadhar, T.C.; et al. Anti-programmed-death-receptor-1 treatment with pembrolizumab in ipilimumab-refractory advanced melanoma: A randomised dose-comparison cohort of a phase 1 trial. Lancet Lond. Engl. 2014, 384, 1109-1117. [CrossRef]

5. Hamid, O.; Robert, C.; Daud, A.; Hodi, F.S.; Hwu, W.J.; Kefford, R.; Wolchok, J.D.; Hersey, P.; Joseph, R.; Weber, J.S.; et al. Five-year survival outcomes for patients with advanced melanoma treated with pembrolizumab in KEYNOTE-001. Ann. Oncol. 2019, 30, 582-588. [CrossRef] [PubMed] 
6. Larkin, J.; Chiarion-Sileni, V.; Gonzalez, R.; Grob, J.J.; Rutkowski, P.; Lao, C.D.; Cowey, C.L.; Schadendorf, D.; Wagstaff, J.; Dummer, R.; et al. Five-Year Survival with Combined Nivolumab and Ipilimumab in Advanced Melanoma. N. Engl. J. Med. 2019, 381, 1535-1546. [CrossRef]

7. Long, G.V.; Atkinson, V.; Cebon, J.S.; Jameson, M.B.; Fitzharris, B.M.; McNeil, C.M.; Hill, A.G.; Ribas, A.; Atkins, M.B.; Thompson, J.A.; et al. Standard-dose pembrolizumab in combination with reduced-dose ipilimumab for patients with advanced melanoma (KEYNOTE-029): An open-label, phase 1b trial. Lancet Oncol. 2017, 18, 1202-1210. [CrossRef]

8. Long, G.V. The anti-PD-1 antibody spartalizumab (S) in combination with dabrafenib (D) and trametinib (T) in previously untreated patients (pts) with advanced BRAF V600-mutant melanoma: Updated efficacy and safety from parts 1 and 2 of COMBI-i. J. Clin. Oncol. 2019, 37, 9531. [CrossRef]

9. Ascierto, P.A.; Ferrucci, P.F.; Fisher, R.; Del Vecchio, M.; Atkinson, V.; Schmidt, H.; Schachter, J.; Queirolo, P.; Long, G.V.; Di Giacomo, A.M.; et al. Dabrafenib, trametinib and pembrolizumab or placebo in BRAF-mutant melanoma. Nat. Med. 2019, 25, 941-946. [CrossRef]

10. Hurwitz, M.E. Baseline tumor-immune signatures associated with response to bempegaldesleukin (NKTR-214) and nivolumab. J. Clin. Oncol. 2019, 37, 2623. [CrossRef]

11. Ribas, A.; Dummer, R.; Puzanov, I.; VanderWalde, A.; Andtbacka, R.H.; Michielin, O.; Olszanski, A.J.; Malvehy, J.; Cebon, J.; Fernandez, E.; et al. Oncolytic Virotherapy Promotes Intratumoral T Cell Infiltration and Improves Anti-PD-1 Immunotherapy. Cell 2017, 170, 1109-1119. [CrossRef] [PubMed]

12. Long, G.V.; Dummer, R.; Andtbacka, R.H. Follow-up analysis of MASTERKEY-265 phase 1b (ph1b) study of talimogene laherparepvec (T-VEC) in combination (combo) with pembrolizumab (pembro) in patients (pts) with unresectable stage IIIB-IVM1c melanoma (MEL). In Society for Melanoma Research Congress; The Society for Melanoma Research: Clifton Park, NY, USA, 2018; pp. 24-27.

13. Ascierto, P.A. Initial efficacy of anti-lymphocyte activation gene-3 (anti-LAG-3; BMS-986016) in combination with nivolumab (nivo) in pts with melanoma (MEL) previously treated with anti-PD-1/PD-L1 therapy. J. Clin. Oncol. 2017, 35, 9520. [CrossRef]

14. Long, G.V.; Dummer, R.; Hamid, O.; Gajewski, T.F.; Caglevic, C.; Dalle, S.; Arance, A.; Carlino, M.S.; Grob, J.J.; Kim, T.M.; et al. Epacadostat plus pembrolizumab versus placebo plus pembrolizumab in patients with unresectable or metastatic melanoma (ECHO-301/KEYNOTE-252): A phase 3, randomised, double-blind study. Lancet Oncol. 2019, 20, 1083-1097. [CrossRef]

15. Hamid, O.; Robert, C.; Daud, A.; Hodi, F.S.; Hwu, W.J.; Kefford, R.; Wolchok, J.D.; Hersey, P.; Joseph, R.W.; Weber, J.S.; et al. Safety and tumor responses with lambrolizumab (anti-PD-1) in melanoma. N. Engl. J. Med. 2013, 369, 134-144. [CrossRef]

16. Gellrich, F.F.; Beissert, S.; Meier, F. Immunotherapy for melanoma. Onkology 2018, 24, 99-103. [CrossRef]

17. Curran, M.A.; Montalvo, W.; Yagita, H.; Allison, J.P. PD-1 and CTLA-4 combination blockade expands infiltrating $\mathrm{T}$ cells and reduces regulatory $\mathrm{T}$ and myeloid cells within B16 melanoma tumors. Proc. Natl. Acad. Sci. USA 2010, 107, 4275-4280. [CrossRef]

18. Winer, A.; Bodor, J.N.; Borghaei, H. Identifying and managing the adverse effects of immune checkpoint blockade. J. Thorac. Dis. 2018, 10, S480-S489. [CrossRef]

19. Long, G.V. 4-year survival and outcomes after cessation of pembrolizumab (pembro) after 2-years in patients (pts) with ipilimumab (ipi)-naive advanced melanoma in KEYNOTE-006. J. Clin. Oncol. 2018, 36, 9503. [CrossRef]

20. Long, G.V.; Stroyakovskiy, D.; Gogas, H.; Levchenko, E.; De Braud, F.; Larkin, J.; Garbe, C.; Jouary, T.; Hauschild, A.; Grob, J.J. Dabrafenib and trametinib versus dabrafenib and placebo for Val600 BRAF-mutant melanoma: A multicentre, double-blind, phase 3 randomised controlled trial. Lancet Lond. Engl. 2015, 386, 444-451. [CrossRef]

21. Nazarian, R.; Shi, H.; Wang, Q.; Kong, X.; Koya, R.C.; Lee, H.; Chen, Z.; Lee, M.K.; Attar, N.; Sazegar, H. Melanomas acquire resistance to B-RAF(V600E) inhibition by RTK or N-RAS upregulation. Nature 2010, 468, 973-977. [CrossRef]

22. Kuske, M.; Westphal, D.; Wehner, R.; Schmitz, M.; Beissert, S.; Praetorius, C.; Meier, F. Immunomodulatory effects of BRAF and MEK inhibitors: Implications for Melanoma therapy. Pharmacol. Res. 2018, 136, 151-159. [CrossRef] [PubMed] 
23. Ribas, A.; Lawrence, D.; Atkinson, V.; Agarwal, S.; Miller, W.H.; Carlino, M.S.; Fisher, R.; Long, G.V.; Hodi, F.S.; Tsoi, J. Combined BRAF and MEK inhibition with PD-1 blockade immunotherapy in BRAF -mutant melanoma. Nat. Med. 2019, 25, 936-940. [CrossRef] [PubMed]

24. Robert, C.; Grob, J.J.; Stroyakovskiy, D.; Karaszewska, B.; Hauschild, A.; Levchenko, E.; Chiarion, V.S.; Schachter, J.; Garbe, C.; Bondarenko, I.; et al. Five-Year Outcomes with Dabrafenib plus Trametinib in Metastatic Melanoma. N. Engl. J. Med. 2019, 381, 626-636. [CrossRef] [PubMed]

25. Bentebibel, S.E.; Hurwitz, M.E.; Bernatchez, C.; Haymaker, C.; Hudgens, C.W.; Kluger, H.M.; Tetzlaff, M.T.; Tagliaferri, M.A.; Zalevsky, J.; Hoch, U.; et al. A First-in-Human Study and Biomarker Analysis of NKTR-214, a Novel IL2R $\beta \gamma$-Biased Cytokine, in Patients with Advanced or Metastatic Solid Tumors. Cancer Discov. 2019, 9, 711-721. [CrossRef] [PubMed]

26. Charych, D.; Khalili, S.; Dixit, V.; Kirk, P.; Chang, T.; Langowski, J.; Rubas, W.; Doberstein, S.K.; Eldon, M.; Hoch, U.; et al. Modeling the receptor pharmacology, pharmacokinetics, and pharmacodynamics of NKTR-214, a kinetically-controlled interleukin-2 (IL2) receptor agonist for cancer immunotherapy. PLoS ONE 2017, 12, e0179431. [CrossRef] [PubMed]

27. Andtbacka, R.H.; Kaufman, H.L.; Collichio, F.; Amatruda, T.; Senzer, N.; Chesney, J.; Delman, K.A.; Spitler, L.E.; Puzanov, I.; Agarwala, S.S.; et al. Talimogene Laherparepvec Improves Durable Response Rate in Patients with Advanced Melanoma. J. Clin. Oncol. 2015, 33, 2780-2788. [CrossRef]

28. Sharma, P.; Allison, J.P. The future of immune checkpoint therapy. Science 2015, 348, 56-61. [CrossRef]

29. NCT02263508. Pembrolizumab with or without Talimogene Laherparepvec or Talimogene Laherparepvec Placebo in Unresected Melanoma (KEYNOTE-034). Available online: https:/clinicaltrials.gov/ct2/show/ NCT02263508 (accessed on 23 August 2019).

30. Ager, C.; Reilley, M.; Nicholas, C.; Bartkowiak, T.; Jaiswal, A.; Curran, M.; Albershardt, T.C.; Bajaj, A.; Archer, J.F.; Reeves, R.S.; et al. 31st Annual Meeting and Associated Programs of the Society for Immunotherapy of Cancer (SITC 2016): Part two. J. Immunother. Cancer 2016, 4, 73. [CrossRef]

31. Mitchell, T.C.; Hamid, O.; Smith, D.C.; Bauer, T.M.; Wasser, J.S.; Olszanski, A.J.; Luke, J.J.; Balmanoukian, A.S.; Schmidt, E.V.; Zhao, Y.; et al. Epacadostat Plus Pembrolizumab in Patients with Advanced Solid Tumors: Phase I Results from a Multicenter, Open-Label Phase I/II Trial (ECHO-202/KEYNOTE-037). J. Clin. Oncol. 2018, 36, 3223. [CrossRef]

32. Blankenstein, S.A.; van Akkooi, A.C. Adjuvant systemic therapy in high-risk melanoma. Melanoma Res. 2019, 29, 358-364. [CrossRef]

33. Gershenwald, J.E.; Scolyer, R.A.; Hess, K.R.; Sondak, V.K.; Long, G.V.; Ross, M.I.; Lazar, A.J.; Faries, M.B.; Kirkwood, J.M.; McArthur, G.A.; et al. Melanoma staging: Evidence-based changes in the American Joint Committee on Cancer eighth edition cancer staging manual. CA Cancer J. Clin. 2017, 67, 472-492. [CrossRef] [PubMed]

34. AEggermont, A.M.; Blank, C.U.; Mandala, M.; Long, G.V.; Atkinson, V.G.; Dalle, S.; Haydon, A.; Lichinitser, M.; Khattak, A.; Carlino, M.S.; et al. Prognostic and predictive value of AJCC-8 staging in the phase III EORTC1325/KEYNOTE-054 trial of pembrolizumab vs placebo in resected high-risk stage III melanoma. Eur. J. Cancer Oxf. Engl. 1990, 116, 148-157. [CrossRef] [PubMed]

35. Eggermont, A.M.; Chiarion-Sileni, V.; Grob, J.J.; Dummer, R.; Wolchok, J.D.; Schmidt, H.; Hamid, O.; Robert, C.; Ascierto, P.A.; Richards, J.M.; et al. Adjuvant ipilimumab versus placebo after complete resection of high-risk stage III melanoma (EORTC 18071): A randomised, double-blind, phase 3 trial. Lancet Oncol. 2015, 16, 522-530. [CrossRef]

36. Eggermont, A.M.; Chiarion-Sileni, V.; Grob, J.J.; Dummer, R.; Wolchok, J.D.; Schmidt, H.; Hamid, O.; Robert, C.; Ascierto, P.A.; Richards, J.M.; et al. Prolonged Survival in Stage III Melanoma with Ipilimumab Adjuvant Therapy. N. Engl. J. Med. 2016, 375, 1845-1855. [CrossRef] [PubMed]

37. Weber, J.; Mandala, M.; Del Vecchio, M.; Gogas, H.J.; Arance, A.M.; Cowey, C.L.; Dalle, S.; Schenker, M.; Chiarion-Sileni, V.; Marquez-Rodas, I.; et al. Adjuvant Nivolumab versus Ipilimumab in Resected Stage III or IV Melanoma. N. Engl. J. Med. 2017, 377, 1824-1835. [CrossRef] [PubMed]

38. Weber, J.S. Adjuvant therapy with nivolumab (NIVO) versus ipilimumab (IPI) after complete resection of stage III/IV melanoma: Updated results from a phase III trial (CheckMate 238). J. Clin. Oncol. 2018, 36, 9502. [CrossRef] 
39. Eggermont, A.M.; Blank, C.U.; Mandala, M.; Long, G.V.; Atkinson, V.; Dalle, S.; Haydon, A.; Lichinitser, M.; Khattak, A.; Carlino, M.S.; et al. Adjuvant Pembrolizumab versus Placebo in Resected Stage III Melanoma. N. Engl. J. Med. 2018, 378, 1789-1801. [CrossRef]

40. Blank, C.U.; Rozeman, E.A.; Menzies, A.M.; van de Wiel, B.A.; Adhikari, C.; Sikorska, K.; Krijgsman, O.; Eriksson, H.; Bierman, C.; Long, G.V.; et al. LBA42OpACIN-neo: A multicenter phase II study to identify the optimal neo-adjuvant combination scheme of ipilimumab (IPI) and nivolumab (NIVO). Ann. Oncol. 2018, 29. [CrossRef]

41. Davies, M.A.; Liu, P.; McIntyre, S.; Kim, K.B.; Papadopoulos, N.; Hwu, W.J.; Hwu, P. Prognostic factors for survival in melanoma patients with brain metastases. Cancer 2011, 117, 1687-1696. [CrossRef]

42. Margolin, K.; Ernstoff, M.S.; Hamid, O.; Lawrence, D.; McDermott, D.; Puzanov, I.; Wolchok, J.D.; Clark, J.I.; Sznol, M.; Logan, T.F.; et al. Ipilimumab in patients with melanoma and brain metastases: An open-label, phase 2 trial. Lancet Oncol. 2012, 13, 459-465. [CrossRef]

43. Long, G.V.; Atkinson, V.; Lo, S.; Sandhu, S.; Guminski, A.D.; Brown, M.P.; Wilmott, J.S.; Edwards, J.; Gonzalez, M.; Scolyer, R.A.; et al. Combination nivolumab and ipilimumab or nivolumab alone in melanoma brain metastases: A multicentre randomised phase 2 study. Lancet Oncol. 2018, 19, 672-681. [CrossRef]

44. Pauline, T.; Clara, A.; Bastien, O.; Stéphane, D.; Laurent, M.; Marie-Thérèse, L.; Bernard, G.; Sophie, D.; Caroline, D.; Jean-Philippe, L.; et al. Impact of radiotherapy administered simultaneously with systemic treatment in patients with melanoma brain metastases within MelBase, a French multicentric prospective cohort. Eur. J. Cancer Oxf. Engl. 1990, 112, 38-46.

45. Tawbi, H.A.; Forsyth, P.A.; Algazi, A.; Hamid, O.; Hodi, F.S.; Moschos, S.J.; Khushalani, N.I.; Lewis, K.; Lao, C.D.; Postow, M.A.; et al. Combined Nivolumab and Ipilimumab in Melanoma Metastatic to the Brain. N. Engl. J. Med. 2018, 379, 722-730. [CrossRef] [PubMed]

46. Tawbi, H.A.H.; Forsyth, P.A.; Hodi, F.S.; Lao, C.D.; Moschos, S.J.; Hamid, O.; Atkins, M.B.; Lewis, K.D.; Thomas, R.P.; Glaspy, J.A.; et al. Efficacy and safety of the combination of nivolumab (NIVO) plus ipilimumab (IPI) in patients with symptomatic melanoma brain metastases (CheckMate 204). J. Clin. Oncol. 2019, 37, 9501. [CrossRef]

47. Hodi, F.S.; Chiarion-Sileni, V.; Gonzalez, R.; Grob, J.J.; Rutkowski, P.; Cowey, C.L.; Lao, C.D.; Schadendorf, D.; Wagstaff, J.; Dummer, R.; et al. Nivolumab plus ipilimumab or nivolumab alone versus ipilimumab alone in advanced melanoma (CheckMate 067): 4-year outcomes of a multicentre, randomised, phase 3 trial. Lancet Oncol. 2018, 19, 1480-1492. [CrossRef]

48. Long, G.V. Long-term Outcomes from the Randomized Ph 2 Study of Nivolumab (nivo) or Nivo+Ipilimumab (ipi) in Patients (pts) with Melanoma Brain Metastases. Ann. Oncol. 2019, 30. [CrossRef]

49. Rauschenberg, R.; Bruns, J.; Brütting, J.; Daubner, D.; Lohaus, F.; Zimmer, L.; Forschner, A.; Zips, D.; Hassel, J.C.; Berking, C.; et al. Impact of radiation, systemic therapy and treatment sequencing on survival of patients with melanoma brain metastases. Eur. J. Cancer 2019, 110, 11-20. [CrossRef]

50. Cao, Y.; Tsien, C.I.; Shen, Z.; Tatro, D.S.; Ten Haken, R.; Kessler, M.L.; Chenevert, T.L. Use of magnetic resonance imaging to assess blood-brain/blood-glioma barrier opening during conformal radiotherapy. J. Clin. Oncol. 2005, 23, 4127-4136. [CrossRef]

51. Twyman-Saint Victor, C.; Rech, A.J.; Maity, A.; Rengan, R.; Pauken, K.E.; Stelekati, E.; Benci, J.L.; Xu, B.; Dada, H.; Odorizzi, P.M.; et al. Radiation and dual checkpoint blockade activate non-redundant immune mechanisms in cancer. Nature 2015, 520, 373-377. [CrossRef]

52. Chicas-Sett, R.; Morales-Orue, I.; Rodriguez-Abreu, D.; Lara-Jimenez, P. Combining radiotherapy and ipilimumab induces clinically relevant radiation-induced abscopal effects in metastatic melanoma patients: A systematic review. Clin. Transl. Radiat. Oncol. 2018, 9, 5-11. [CrossRef]

53. Blum, E.S.; Yang, J.; Komatsubara, K.M.; Carvajal, R.D. Clinical Management of Uveal and Conjunctival Melanoma. Oncol. Williston Park N 2016, 30, 29-43.

54. Van Raamsdonk, C.D.; Bezrookove, V.; Green, G.; Bauer, J.; Gaugler, L.; O’Brien, J.M.; Simpson, E.M.; Barsh, G.S.; Bastian, B.C. Frequent somatic mutations of GNAQ in uveal melanoma and blue naevi. Nature 2009, 457, 599-602. [CrossRef] [PubMed]

55. Van Raamsdonk, C.D.; Griewank, K.G.; Crosby, M.B.; Garrido, M.C.; Vemula, S.; Wiesner, T.; Obenauf, A.C.; Wackernagel, W.; Green, G.; Bouvier, N.; et al. Mutations in GNA11 in uveal melanoma. N. Engl. J. Med. 2010, 363, 2191-2199. [CrossRef] [PubMed] 
56. Refaian, N.; Schlereth, S.L.; Koch, K.R.; Notara, M.; Hos, D.; Mescher, M.; Iden, S.; Bosch, J.J.; Jager, M.J.; Cursiefen, C.; et al. Comparing the Hem- and Lymphangiogenic Profile of Conjunctival and Uveal Melanoma Cell Lines. Investig. Ophthalmol. Vis. Sci. 2015, 56, 5691-5697. [CrossRef]

57. Mignard, C.; Deschamps Huvier, A.; Gillibert, A.; Duval Modeste, A.B.; Dutriaux, C.; Khammari, A.; Avril, M.F.; Kramkimel, N.; Mortier, L.; Marcant, P.; et al. Efficacy of Immunotherapy in Patients with Metastatic Mucosal or Uveal Melanoma. J. Oncol. 2018, 2018, 1908065. [CrossRef]

58. Heppt, M.V.; Heinzerling, L.; Kähler, K.C.; Forschner, A.; Kirchberger, M.C.; Loquai, C.; Meissner, M.; Meier, F.; Terheyden, P.; Schell, B.; et al. Prognostic factors and outcomes in metastatic uveal melanoma treated with programmed cell death-1 or combined PD-1/cytotoxic T-lymphocyte antigen-4 inhibition. Eur. J. Cancer Oxf. Engl. 1990, 82, 56-65. [CrossRef]

59. Pelster, M. Phase II study of ipilimumab and nivolumab (ipi/nivo) in metastatic uveal melanoma (UM). J. Clin. Oncol. 2019, 37. [CrossRef]

60. McLaughlin, C.C.; Wu, X.-C.; Jemal, A.; Martin, H.J.; Roche, L.M.; Chen, V.W. Incidence of noncutaneous melanomas in the U.S. Cancer 2005, 103, 1000-1007. [CrossRef]

61. Heppt, M.V.; Roesch, A.; Weide, B.; Gutzmer, R.; Meier, F.; Loquai, C.; Kähler, K.C.; Gesierich, A.; Meissner, M.; von Bubnoff, D.; et al. Prognostic factors and treatment outcomes in 444 patients with mucosal melanoma. Eur. J. Cancer Oxf. Engl. 1990, 81, 36-44. [CrossRef]

62. Curtin, J.A.; Fridlyand, J.; Kageshita, T.; Patel, H.N.; Busam, K.J.; Kutzner, H.; Cho, K.H.; Aiba, S.; Bröcker, E.B.; LeBoit, P.E.; et al. Distinct sets of genetic alterations in melanoma. N. Engl. J. Med. 2005, 353, 2135-2147. [CrossRef]

63. D’Angelo, S.P.; Larkin, J.; Sosman, J.A.; Lebbé, C.; Brady, B.; Neyns, B.; Schmidt, H.; Hassel, J.C.; Hodi, F.S.; Lorigan, P.; et al. Efficacy and Safety of Nivolumab Alone or in Combination with Ipilimumab in Patients with Mucosal Melanoma: A Pooled Analysis. J. Clin. Oncol. 2017, 35, 226-235. [CrossRef] [PubMed]

64. Furney, S.J.; Turajlic, S.; Stamp, G.; Thomas, J.M.; Hayes, A.; Strauss, D.; Gavrielides, M.; Xing, W.; Gore, M.; Larkin, J.; et al. The mutational burden of acral melanoma revealed by whole-genome sequencing and comparative analysis. Pigment Cell Melanoma Res. 2014, 27, 835-838. [CrossRef] [PubMed]

65. Bello, D.M.; Chou, J.F.; Panagea, K.S.; Brady, M.S.; Coit, D.G.; Carvajal, R.D. Prognosis of Acral Melanoma: A Series of 281 Patients. Ann. Surg. Oncol. 2013, 20, 3618-3625. [CrossRef] [PubMed]

66. Shoushtari, A.N.; Munhoz, R.R.; Kuk, D.; Ott, P.A.; Johnson, D.B.; Tsai, K.K.; Rapisuwon, S.; Eroglu, Z.; Sullivan, R.J.; Luke, J.J.; et al. The efficacy of anti-PD-1 agents in acral and mucosal melanoma. Cancer 2016, 122, 3354-3362. [CrossRef]

67. Shain, A.H.; Garrido, M.; Botton, T.; Talevich, E.; Yeh, I.; Sanborn, J.Z.; Chung, J.; Wang, N.J.; Kakavand, H.; Mann, G.J.; et al. Exome sequencing of desmoplastic melanoma identifies recurrent NFKBIE promoter mutations and diverse activating mutations in the MAPK pathway. Nat. Genet. 2015, 47, 1194-1199. [CrossRef]

68. Eroglu, Z.; Zaretsky, J.M.; Hu-Lieskovan, S.; Kim, D.W.; Algazi, A.; Johnson, D.B.; Liniker, E.; Kong, B.; Munhoz, R.; Rapisuwon, S.; et al. High response rate to PD-1 blockade in desmoplastic melanomas. Nature 2018, 553, 347-350. [CrossRef]

69. Larkin, J.; Chiarion-Sileni, V.; Gonzalez, R.; Grob, J.J.; Cowey, C.L.; Lao, C.D.; Schadendorf, D.; Dummer, R.; Smylie, M.; Rutkowski, P.; et al. Combined Nivolumab and Ipilimumab or Monotherapy in Previously Untreated Melanoma. N. Engl. J. Med. 2015, 373, 23-34. [CrossRef]

70. Brahmer, J.R.; Lacchetti, C.; Schneider, B.J.; Atkins, M.B.; Brassil, K.J.; Caterino, J.M.; Chau, I.; Ernstoff, M.S.; Gardner, J.M.; Ginex, P.; et al. Management of Immune-Related Adverse Events in Patients Treated with Immune Checkpoint Inhibitor Therapy: American Society of Clinical Oncology Clinical Practice Guideline. J. Clin. Oncol. Off. J. Am. Soc. Clin. Oncol. 2018, 36, 1714-1768. [CrossRef]

71. Perez-Ruiz, E.; Minute, L.; Otano, I.; Alvarez, M.; Ochoa, M.C.; Belsue, V.; de Andrea, C.; Rodriguez-Ruiz, M.E.; Perez-Gracia, J.L.; Marquez-Rodas, I.; et al. Prophylactic TNF blockade uncouples efficacy and toxicity in dual CTLA-4 and PD-1 immunotherapy. Nature 2019, 569, 428-432. [CrossRef]

(C) 2020 by the authors. Licensee MDPI, Basel, Switzerland. This article is an open access article distributed under the terms and conditions of the Creative Commons Attribution (CC BY) license (http://creativecommons.org/licenses/by/4.0/). 